\title{
POWER SPECTRUM OPTIMIZATION IN THE DESIGN OF MULTISINE MANOEUVRE FOR IDENTIFICATION PURPOSES
}

\author{
Piotr Lichota, JoAnna SzulczyK \\ Warsaw University of Technology, Institute of Aeronautics and Applied Mechanics, Poland \\ e-mail: plichota@meil.pw.edu.pl; jszulczyk@meil.pw.edu.pl \\ Daniel Agudelo Noreña, Felipe A. Vallejo Monsalve \\ Universidad de San Buenaventura, Bogotá, Colombia \\ e-mail: dagudelon@gmail.com; fvallejo@usbbog.edu.co
}

\begin{abstract}
In this paper, two sets of multisine signals are designed for system identification purposes. The first one is obtained without any information about system dynamics. In the second case, the a priori information is given in terms of dimensional stability and control derivatives. Magnitude Bode plots are obtained to design the multisine power spectrum that is optimized afterwards. A genetic algorithm with linear ranking, uniform crossover and mutation operator has been employed for that purpose. Both designed manoeuvres are used to excite the aircraft model, and then system identification is performed. The estimated parameters are obtained by applying two methods: Equation Error and Output Error. The comparison of both investigated cases in terms of accuracy and manoeuvre time is presented afterwards.
\end{abstract}

Keywords: flight dynamics, optimal input design, multisine excitations, system identification, least squares, maximum likelihood

\section{Introduction}

System identification (Sys-ID) techniques play an important role in the development of high-quality aircraft-simulation models from flight-test data (Grauer, 2016; Albisser et al., 2017; Viana, 2016). The main purpose of those approaches is to develop a mathematical model of the aircraft. To achieve this aim, an adequate input has to be designed in order to obtain dynamic characteristics of the object from flight tests.

The research on this topic started in the 1960s when Levin (1960) made the first systematic attempt at obtaining optimal inputs. Later, in the late 60s, Nahi and Wallis (1969) made a significant step by using an optimality criterion to design inputs in the time domain. Since then the use of optimality criteria was a great deal of interest and was investigated by, e.g., Fedorov (1972), Kalaba and Springarn (1982) or Goodwin and Payne (1997). At first, designed inputs were used for manoeuvres in which only one flight control was deflected. Further investigation made by, e.g., Wells and Ramachadran (1977) or Morelli and Klein (1990) showed that designing sets of excitations in which flight-control surfaces were deflected simultaneously was also possible. Even though, none of the theoretical developments seemed to be fully adapted for solving complex issues. Some recent progress has been made in applying numerical algorithms and incorporating them with other tools to design optimal inputs (Seren et al., 2006, 2013; Lichota, 2016; Lichota et al., 2017).

To shorten the time of the flight test campaign, multisine excitations that allow simultaneous flight control deflections can be used. Planning manoeuvres with those inputs requires the designing of their power spectrum. In our study, a genetic algorithm has been used to optimize 
that energy distribution. The main purpose is to improve the input effectiveness by maximizing the power stored in certain harmonics.

The organization of this article is as follows. First, we describe the process of designing multisine signals for simultaneous aileron and elevator deflections. That section is divided into two parts. In the first one, a method for designing the inputs without a priori knowledge of the object is presented. In the second part, the optimization process (based on the available information of the aircraft dynamics) that leads to obtaining energy optimized multisine excitations is described. Parameter estimation methods used for estimating stability and control derivatives from the model response are presented in the next Section. Comparison of inputs in terms of manoeuvre time and Sys-ID accuracy is shown thereafter. The paper closes with a short summary of conclusions.

\section{Multisine input design}

One of the approaches that allows one to shorten the time spent during flight tests in order to reduce the total cost of the Sys-ID process is to design a manoeuvre with simultaneous flight control deflections. Multisine input signals that are mutually orthogonal in the time and frequency domain can be easily designed for those purposes. To achieve this aim different harmonics need to be assigned to each flight control (Morelli, 2009)

$$
u=\sum_{k=1}^{M} A_{k} \sin \left(2 \pi f_{k} t+\phi_{k}\right)
$$

where $f_{k}$ are consecutive harmonic frequencies, $A_{k}$ is the amplitude of $k$-th harmonic, $\phi_{k}$ is the phase shift angle and $t$ denotes time.

\subsection{Uniform power spectrum}

When there is no knowledge about the aircraft dynamics, it should be assumed that the same amount of information can be obtained from each power spectrum component. Therefore, the amplitude of each harmonic is designed for the energy content that is uniformly distributed along the frequency range of interest

$$
A_{j, k}=A_{j} \sqrt{p_{j, k}}
$$

where $p_{j, k}$ is the power of the $k$-th harmonic component assigned to the $j$-th flight control.

To increase the efficiency of the input signal, the phase shift angles $\phi_{k}$ have to be adjusted by minimizing the Relative Peak Factor

$$
R P F\left(u_{j}\right)=\frac{\max \left(u_{j}\right)-\min \left(u_{j}\right)}{2 \sqrt{2} r m s\left(u_{j}\right)}
$$

Initial values of the phase angles are obtained by using the Schroeder formula (Schroeder, 1970)

$$
\phi_{j, k}=\phi_{j, k-1}+2 \pi\left(f_{j, k-1}-f_{j, k}\right) \frac{k-1}{M_{j}} T
$$

The Nelder-Mead simplex algorithm (Lagarias et al., 1998) has been used to minimize the non-linear cost function.

In order to obtain non-corellated inputs, even harmonics have been assigned to the elevator and the rest of the set to the ailerons. The first harmonic is omitted due to signal optimization which is performed later, therefore the lowest frequency is $0.1 \mathrm{~Hz}$. The total set is sampled with a resolution of $0.05 \mathrm{~Hz}$ and the upper bound of the frequency range of interest is set to $2 \mathrm{~Hz}$. Moreover, to start and finish the control vector with zero amplitude, each input signal is shifted along the time axis. As a result, we obtain a control vector $\mathbf{u}$ shown in Fig. 1. 

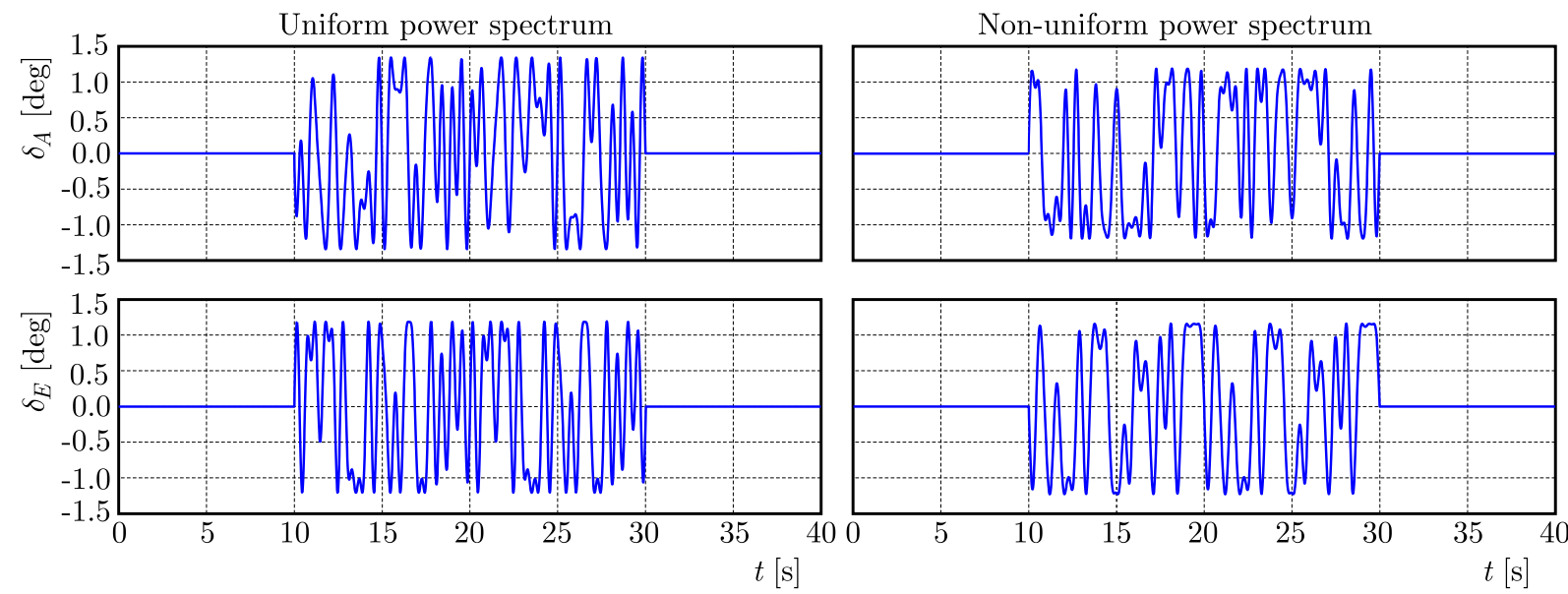

Fig. 1. Multisine input signals

\subsection{Non-uniform power spectrum}

In the second case, the multisine inputs are designed by including the a priori knowledge of the system dynamics. The initial information is given in terms of dimensional stability and control derivatives. Improvement of the signals efficiency is achieved by using the a priori knowledge in order to design a power spectrum with the energy that is maximized at significant frequencies. To select the frequencies at which more power should be stored, the magnitude Bode plots for all aerodynamic force and moment components are created. One of the Bode plots used for the reference power spectrum design is presented in Fig. 2.

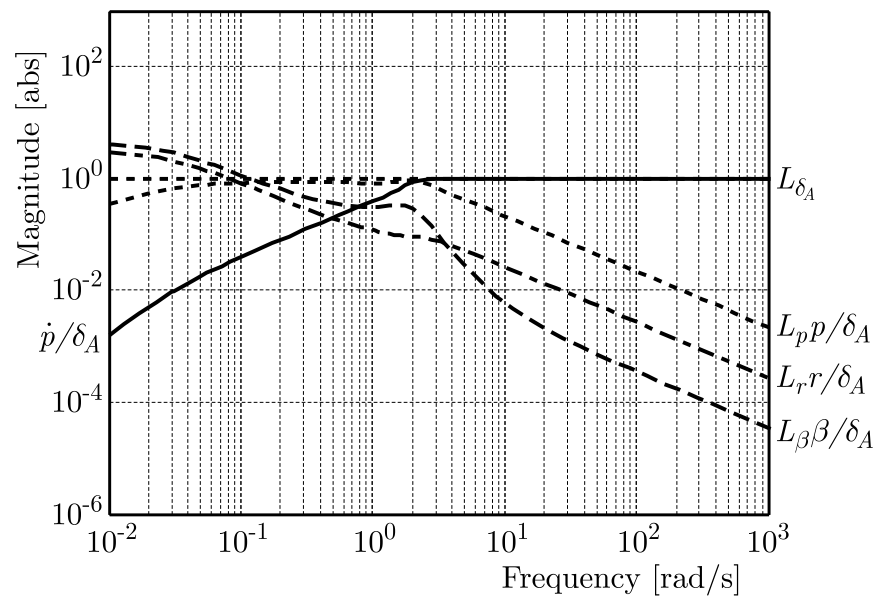

Fig. 2. Rolling moment Bode plot for aileron deflection

The reference power spectrum designed on the Bode plots base and Marchand method (Marchand, 1974) is showed in Fig. 3.

In order to optimize the design, the power stored at different harmonics is adjusted by introducing weighting factors so that the energy distribution minimizing the $R P F$ maximizes the input efficiency.

A genetic algorithm with linear ranking, uniform crossing and 5\% mutation probability has been used to obtain the final values of the weighting factors. We chose the genetic algorithm for optimization because the cost function has multiple local minima and the search space is large. Due to mutual orthogonality of the multisine inputs, this procedure is performed separately for each control surface. The obtained optimized power spectrum shown in Fig. 4 is used later to design the control vector $\mathbf{u}$ that is presented in Fig. 1. 


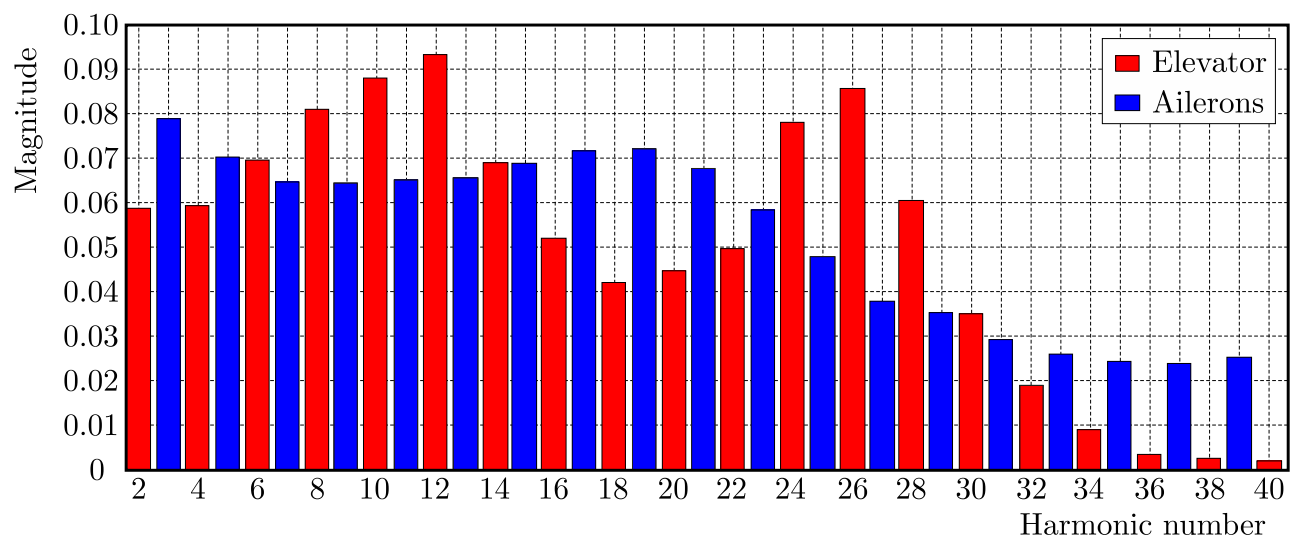

Fig. 3. Reference Power Spectrum

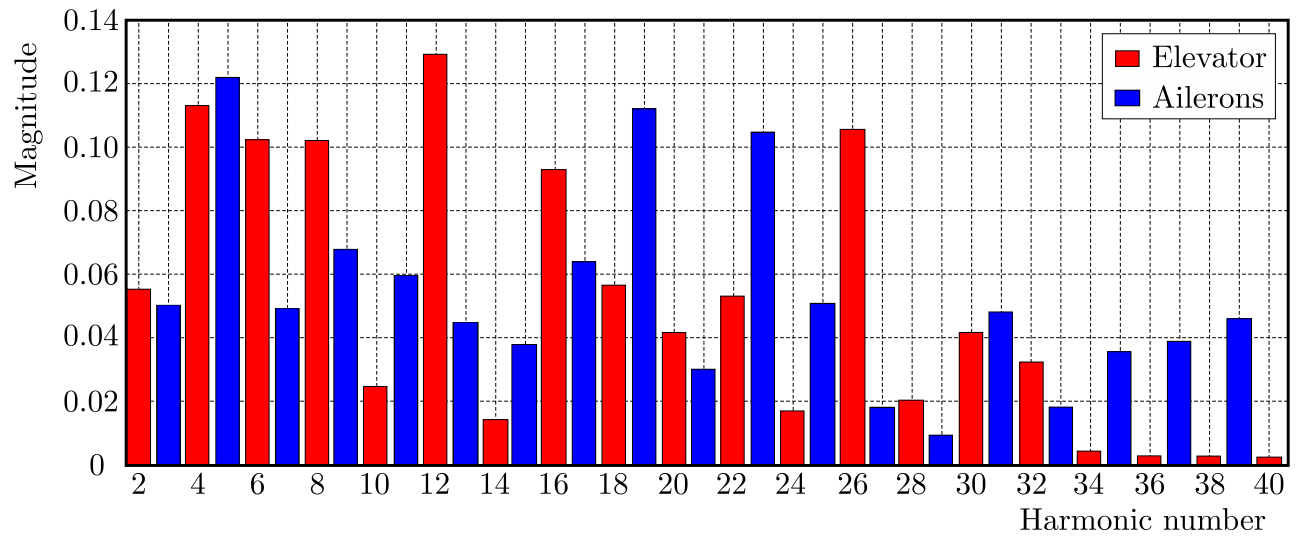

Fig. 4. Optimized Power Spectrum

\section{System identification}

The general aim of the parametric Sys-ID is to determine an adequate mathematical model which contains unknown values of certain system coefficients that have to be obtained indirectly from measured data. To achieve this goal, specific experiments have to be done in order to excite the system sufficiently and register inputs and object responses. From the modelling aspect, it means that determined equations must provide the model response $\mathbf{y}$ which matches adequately the measured system response $\mathbf{z}$.

In this paper, to perform the Sys-ID experiment, simultaneous multsine inputs for both investigated cases are used as excitations for a transport aircraft model with cross-coupling derivatives. To perform parameter estimation, the input signals (elevator and ailerons deflections) and aircraft response (longitudinal velocity, angle of attack, sideslip angle, roll, pitch, yaw rates, roll and pitch angles and linear acceleration components) are recorded. As the signals are corrupted with measurement noise, a 15-th point low-pass digital filter is used (Kendall et al., 1983)

$$
\begin{aligned}
y_{n}= & \frac{1}{320}\left(-3 u_{n-7}-6 u_{n-6}-5 u_{n-5}+3 u_{n-4}+21 u_{n-3}+46 u_{n-2}+67 u_{n-1}+74 u_{n}\right. \\
& \left.+67 u_{n+1}+46 u_{n+2}+21 u_{n+3}+3 u_{n+4}-5 u_{n+5}-6 u_{n+6}-3 u_{n+7}\right)
\end{aligned}
$$

\subsection{Equation Error Method}

Multiple different approaches can be used to perform the aircraft Sys-ID, e.g., Equation Error Methods, Output Error Methods, Filter Error Methods or Artificial Neural Networks. Equation 
Error Methods minimize the cost function defined directly by the input-output equation. Among this class, the Ordinary Least Squares (OLS) is the most popular due to its mathematical simplicity

$$
\mathbf{Y}=\mathbf{X} \Theta+\epsilon
$$

where $\mathbf{Y}$ are dependent variables, $\boldsymbol{\Theta}$ denotes unknown parameters, $\boldsymbol{\epsilon}$ represent equation errors and $\mathbf{X}$ are independent variables. To obtain the estimates, the cost function which is the sum of the squares of the residuals is minimized

$$
J(\Theta)=\frac{1}{2} \sum_{k=1}^{N} \epsilon^{2}(k)=\frac{1}{2}[\mathbf{Y}-\mathbf{X} \Theta]^{\mathrm{T}}[\mathbf{Y}-\mathbf{X} \Theta]
$$

where $k$ are discrete time points indices.

As a result, the estimates of the unknown parameters are given by

$$
\widehat{\mathbf{\Theta}}=\left(\mathbf{X}^{\mathrm{T}} \mathbf{X}\right)^{-1} \mathbf{X}^{\mathrm{T}} \mathbf{Y}
$$

The block schematic of the OLS is presented in Fig. 5 .

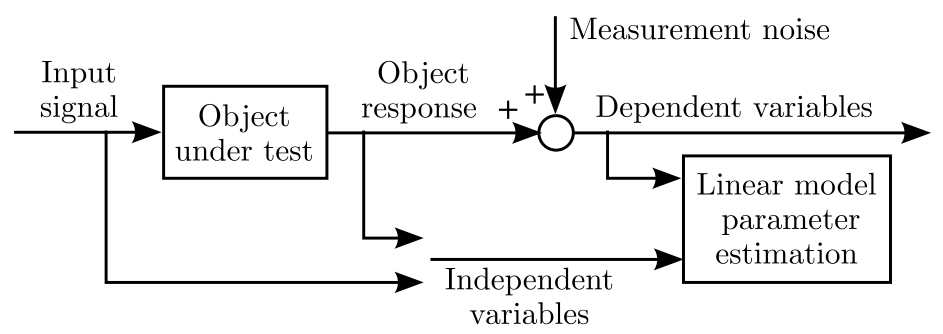

Fig. 5. Block schematic of the Least Squares Method

Before using the OLS to estimate stability and control derivatives, a data preprocessing step is performed. It is required to differentiate selected measured object responses and eliminate biases from the signals. To increase the accuracy of the estimates by eliminating parameters that have no physical meaning, Eq. (3.2) is solved independently for each output signal. In the parameter estimation process, the backward elimination is used to determine the model structure.

\subsection{Output Error Method}

After performing the Sys-ID using the OLS it turned out that the obtained results are not satisfying. Regardless of the excitations set, the absolute relative error is considered too high for $N_{r}$. When the identification experiment is designed without a priori knowledge $M_{u}, M_{q}$ and $N_{\beta}$ are estimated with large uncertainty. When the a priori information is included, it is observed for $N_{p}$.

As the estimates are biased due to measurement noise in the registered signals, to increase accuracy of the estimates, the Output Error Method (OEM) is used. This approach is the most widely applied Sys-ID technique. The main reason for its popularity is its good representation of a natural formulation for a dynamic system. The block schematic of the OEM is shown in Fig. 6.

The OEM used in this study is based on the Maximum Likelihood Estimation (MLE), therefore it seeks for maximizing the probability of observing the measured responses for model parameters (Jategaonkar, 2015)

$$
\widehat{\boldsymbol{\Theta}}=\arg \max _{\boldsymbol{\Theta}} p(\mathbf{z} \mid \boldsymbol{\Theta})
$$




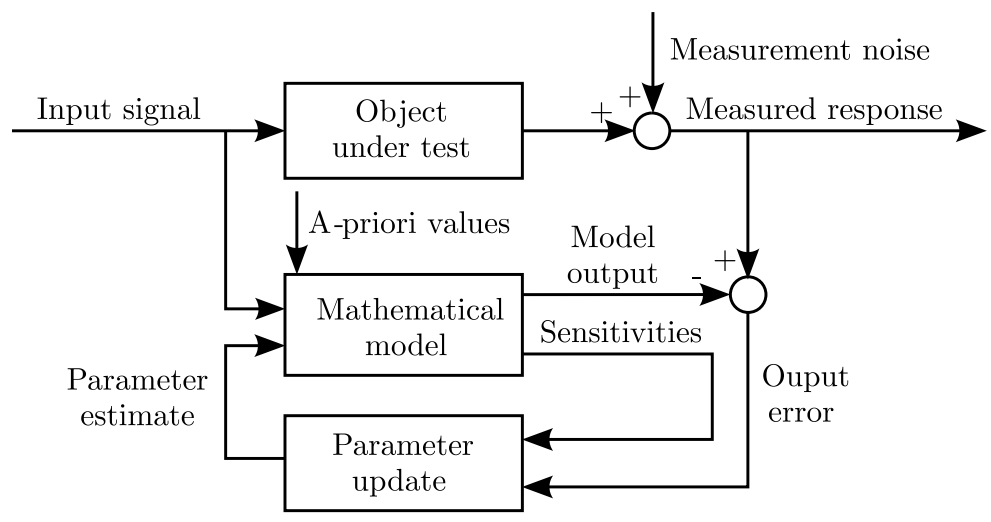

Fig. 6. Block schematic of the Output Error Method

where $\mathbf{z}$ denotes the measured response, $\boldsymbol{\Theta}$ are model parameters and the hat symbol stands for the estimates. To obtain the estimates, the cost function which is negative log-likelihood, has been minimized

$$
\mathcal{L}(\mathbf{\Theta} \mid \mathbf{z})=\frac{1}{2} \sum_{k=1}^{N}\left[\mathbf{z}\left(t_{k}\right)-\mathbf{y}\left(t_{k}\right)\right]^{\mathrm{T}} \mathbf{R}^{-1}\left[\mathbf{z}\left(t_{k}\right)-\mathbf{y}\left(t_{k}\right)\right]+\frac{n N}{2} \ln (2 \pi)+\frac{N}{2} \ln (\operatorname{det}(\mathbf{R}))
$$

where $t_{k}$ is the discrete time at the $k$-th point, $k=1, \ldots, N, \mathbf{R}$ is the measurement noise covariance matrix and $n$ denotes the number of model outputs. The unknown measurement covariance matrix $\mathbf{R}$ is estimated from

$$
\widehat{\mathbf{R}}=\frac{1}{N} \sum_{k=1}^{N}\left[\mathbf{z}\left(t_{k}\right)-\mathbf{y}\left(t_{k}\right)\right]\left[\mathbf{z}\left(t_{k}\right)-\mathbf{y}\left(t_{k}\right)\right]^{\mathrm{T}}
$$

Substituting the measurement covariance matrix into Eq. (3.6) and neglecting the constant terms allows one to simplify the cost function to the form given by

$$
J(\Theta)=\prod_{l=1}^{m} \frac{1}{N} \sum_{k=1}^{N}\left(z_{i}\left(t_{k}\right)-y_{i}\left(t_{k}\right)\right)^{2}
$$

where $l=1, \ldots, m$ is the number of system outputs.

Similarly to the OLS, when the MLE is applied, backward elimination is used to determine the model structure.

\section{Results}

To determine if including the a priori knowledge in the multisine inputs design improves the identification experiment, several criteria are used. For that purpose, deviations from the trim condition, magnitude coherence function and estimates accuracy are investigated.

\subsection{Manoeuvre analysis}

To increase the model efficiency designed manoeuvres should allow the aircraft to remain close to the equilibrium point in which it is excited, and the time spent to perform a flight experiment should be minimized. Small deviations from the trim conditions decrease the stabilization time, so the next manoeuvre can be performed faster.

Deviations of various flight parameters from the equilibrium point for both investigated cases are presented in Fig. 7. From the cross-plot, it can be seen that the dispersion of the motion 
parameters from their initial values are slightly smaller for the uniform power spectrum. It leads to a conclusion that designing the inputs by only optimizing their power spectrum does not result in shortening the manoeuvre time. Nevertheless, the obtained deviations from the trim point are small for both cases. This means that both designs are short in time in comparison to the conventional approach, and even the global aerodynamic parameter estimation can be performed.
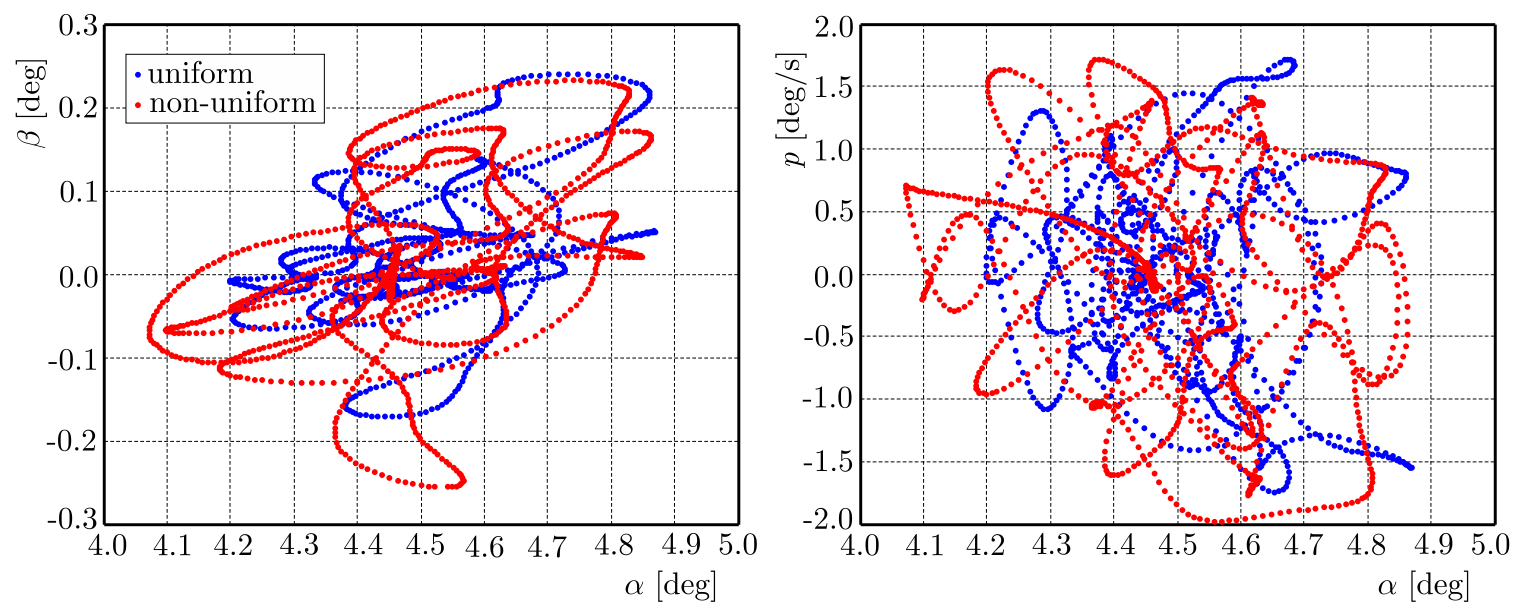

Fig. 7. Cross-plots of flight parameters

To asses the amount of information that can be obtained from a specific manoeuvre, the magnitude squared coherence function (known as coherence) can be used (Young and Patton, 1988 ) as it is a measure of the linear dependence between the input and output signals

$$
\Gamma_{x y}^{2}\left(f_{k}\right)=\frac{\left|G_{x y}\left(f_{k}\right)\right|^{2}}{G_{x x}\left(f_{k}\right) G_{y y}\left(f_{k}\right)}
$$

where $G_{x y}, G_{x x}, G_{y y}$ are cross, input and output spectral densities.

When there is an ideal linear dependency between the input and output signal, the coherence equals 1 , whilst 0 means that there is no dependence between those signals. Typically, a value above 0.6 provides a good object response mapping for multi-output systems (Tischler and Remple, 2012).

Coherence for the roll rate due to ailerons and pitch rate due to elevator deflection for both investigated designs is shown in Fig. 8. In our study, we observed that the coherence obtained for both manoeuvres is comparable. As it can be seen from the plots, the coherence is almost 1 in the whole frequency range when the elevator is deflected, and over 0.6 when the ailerons are used. For the roll rate due to ailerons deflection, the design with the a priori knowledge is slightly better in the low frequency range, however both manoeuvres provide sufficient amount of information as the coherence is above the assumed threshold.

\subsection{Estimates accuracy}

After the whole data set containing excitations (aileron $\delta_{A}$ and elevator deflections $\delta_{E}$ ) and model response (longitudinal velocity $u$, angle of attack $\alpha$, sideslip angle $\beta$, angular rates $p$, $q, r$, attitude angles $\phi, \theta$ and linear acceleration components $a_{x}, a_{y}, a_{z}$ ) has been collected and filtered, Sys-ID is performed. The OLS are used for that purpose. To obtain a mathematical model of the object, the recorded signals are preprocessed and the backward elimination is used to determine the model structure. To compare the results obtained for both manoeuvres (uniform and non-uniform power spectrum), an absolute relative error for each aerodynamic coefficient is evaluated. The obtained outcomes are presented in Table 1. 

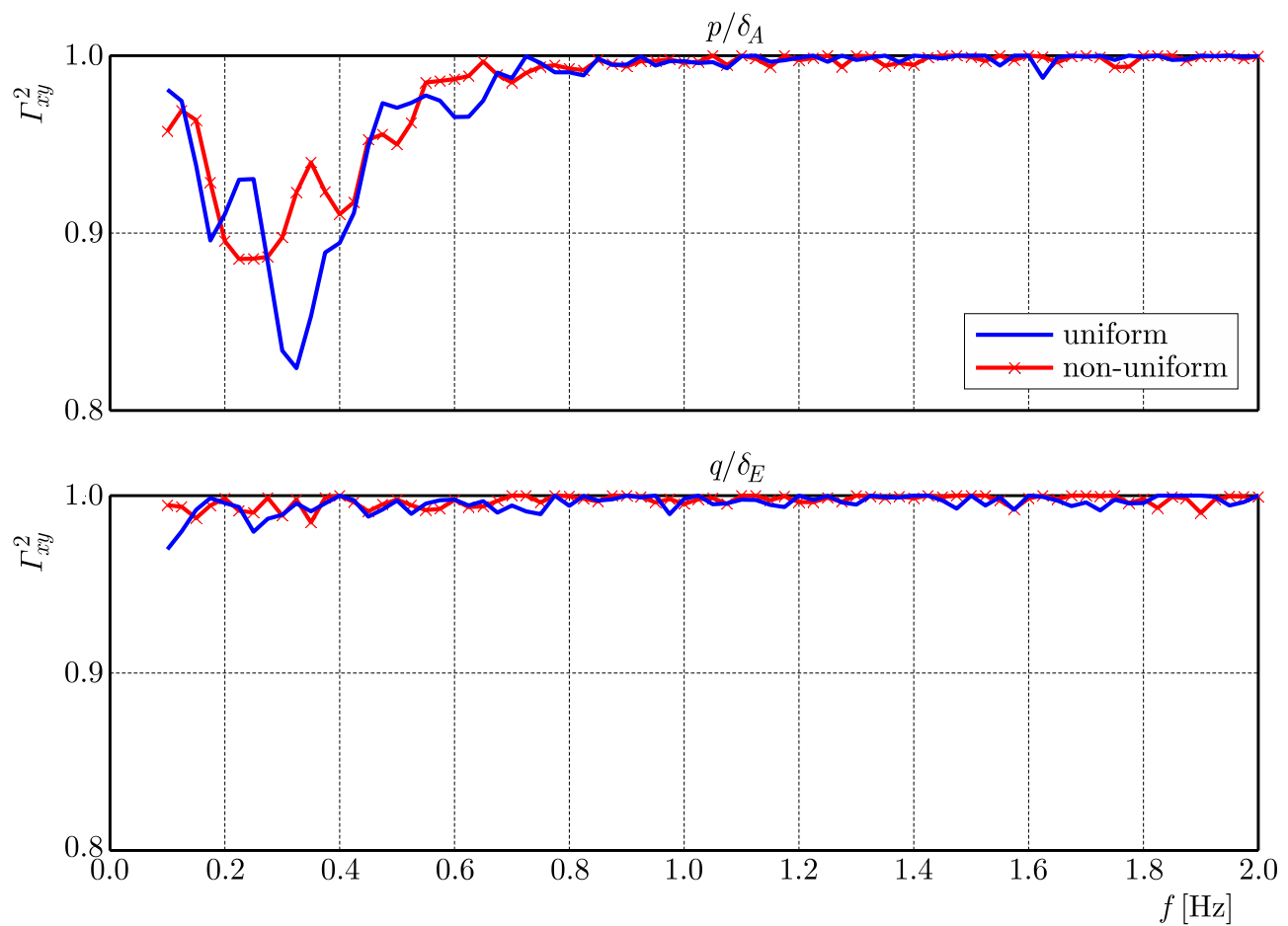

Fig. 8. Coherence function

Table 1. Absolute relative error for Equation Error Method, [\%]

\begin{tabular}{|c|c|c|c|c|c|}
\hline Name & Uniform & Optimized & Name & Uniform & Optimized \\
\hline \hline$X_{\alpha}$ & 2.74 & 1.35 & $L_{\beta}$ & 2.03 & 2.89 \\
\hline$Z_{\alpha}$ & 5.07 & 5.42 & $L_{p}$ & 7.33 & 4.55 \\
\hline$M_{u}$ & 16.29 & 8.83 & $L_{r}$ & 3.64 & 1.42 \\
\hline$M_{\alpha}$ & 6.13 & 3.68 & $L_{\delta_{A}}$ & 1.14 & 0.83 \\
\hline$M_{q}$ & 13.31 & 8.58 & $N_{\alpha}$ & 0.06 & 1.23 \\
\hline$M_{\delta_{E}}$ & 2.26 & 1.72 & $N_{\beta}$ & 10.51 & 6.23 \\
\hline$Y_{\beta}$ & 0.15 & 0.36 & $N_{p}$ & 6.29 & 14.47 \\
\hline$Y_{p}$ & 0.23 & 0.03 & $N_{r}$ & 31.35 & 34.18 \\
\hline$Y_{r}$ & 1.09 & 0.02 & $N_{\delta_{A}}$ & 2.88 & 4.13 \\
\hline$Y_{\delta_{A}}$ & 0.03 & 0.05 & & & \\
\hline
\end{tabular}

From Table 1 it can be observed that the absolute relative error values are high for both investigated cases. It could be caused by the fact that there is a measurement noise in the registered signals. As it was mentioned earlier, the Sys-ID is performed again using a more sophisticated method, which is the MLE, because it allows one to include the measurement noise. The outcomes are presented in Table. 2.

Table 2 shows that the parameters are estimated with high accuracy in both cases. It can be seen that the non-uniform power spectrum allows one to evaluate slightly better the outcomes. On the other hand, one has to have in mind that for a non-uniform case, additional computational time has to be spent to perform power spectrum optimization. The results of the Sys-ID performed using the MLE in form of lateral and longitudinal acceleration time histories are presented in Fig. 9.

In the plots, the recorded object response obtained for both sets of the input signals is represented by blue lines, whilst the red lines with black markers show the results of the Sys-ID process. Almost an ideal match between the measurements for all flight parameters is observed 
Table 2. Absolute relative error for Output Error Method, [\%]

\begin{tabular}{|c|c|c|c|c|c|}
\hline Name & Uniform & Optimized & Name & Uniform & Optimized \\
\hline \hline$X_{\alpha}$ & 0.3346 & 0.2409 & $L_{\alpha}$ & 1.8495 & 1.8454 \\
\hline$Z_{\alpha}$ & 0.3134 & 0.2233 & $L_{\beta}$ & 0.1540 & 0.0573 \\
\hline$Z_{\delta_{E}}$ & 2.1896 & 0.8917 & $L_{p}$ & 0.1689 & 0.1440 \\
\hline$M_{u}$ & 0.5759 & 0.1646 & $L_{r}$ & 0.3220 & 0.0698 \\
\hline$M_{\alpha}$ & 0.0598 & 0.1160 & $L_{\delta_{A}}$ & 0.1409 & 0.1221 \\
\hline$M_{q}$ & 0.3982 & 0.0529 & $N_{\alpha}$ & 0.0205 & 0.0304 \\
\hline$M_{\delta_{E}}$ & 0.2597 & 0.0846 & $N_{\beta}$ & 0.0369 & 0.0471 \\
\hline$Y_{\beta}$ & 0.0568 & 0.0228 & $N_{p}$ & 0.2287 & 0.0540 \\
\hline$Y_{p}$ & 0.2089 & 0.3757 & $N_{r}$ & 0.0329 & 0.0748 \\
\hline$Y_{r}$ & 0.1619 & 0.0261 & $N_{\delta_{R}}$ & 0.2930 & 0.0426 \\
\hline$Y_{\delta_{R}}$ & 0.3917 & 0.3380 & & & \\
\hline
\end{tabular}
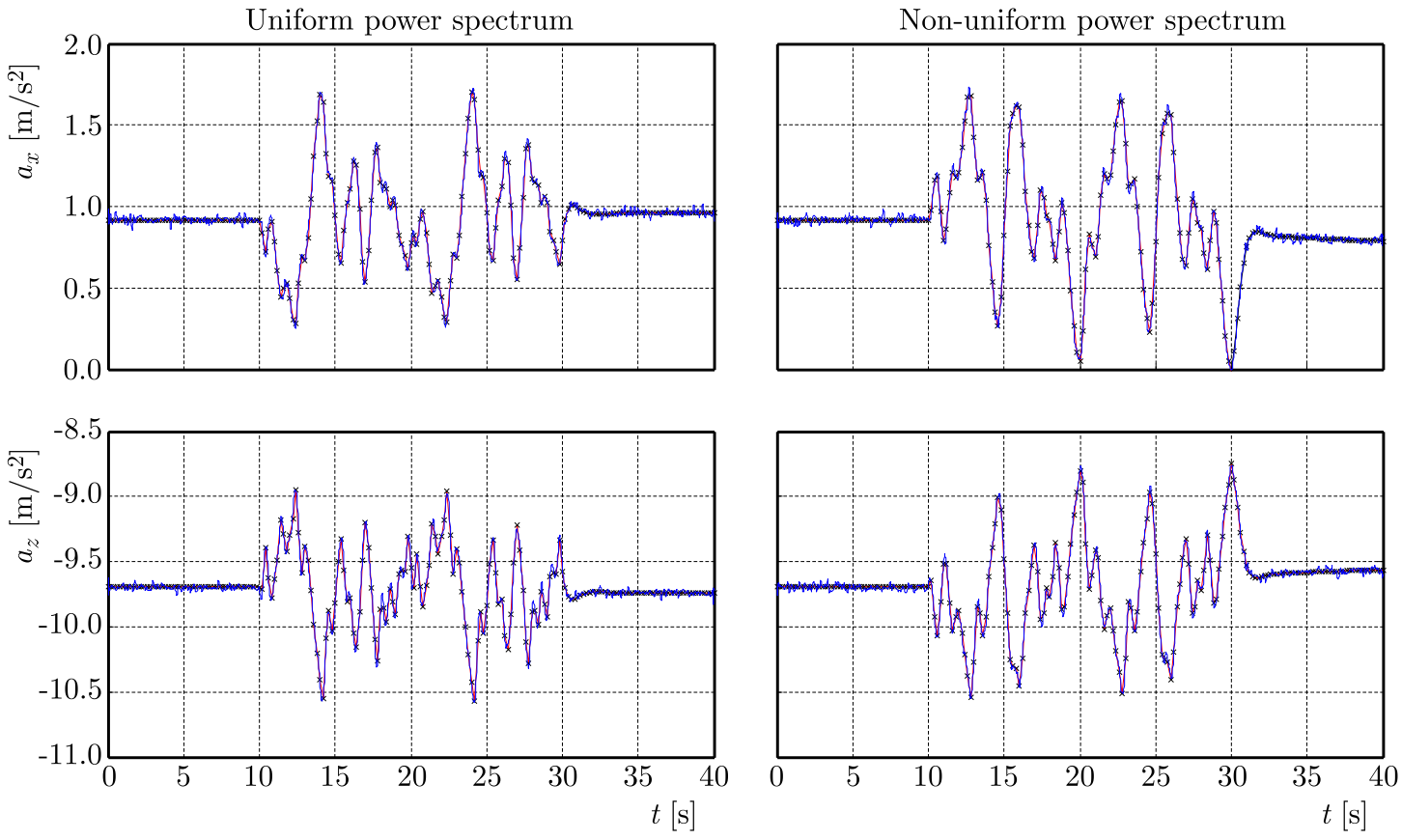

Fig. 9. Time histories for MLE

for both cases. The flight parameters time history plots confirm also our previous conclusion regarding the stabilization time, i.e. when the uniform power spectrum has been used, the aircraft returned to the trim point slightly faster. Moreover, if the non-uniform energy distribution has been selected, a new equilibrium point was reached.

\section{Conclusions}

In this paper, it is shown how to design multisine input signals with a priori information of the system dynamics in order to improve the Sys-ID accuracy. Two types of multisine excitations are used in our investigation.

In the first case, the input signals are obtained without any information of the aircraft dynamics. However, for the majority of Sys-ID tests, there is an a priori knowledge of aerodynamic coefficients provided that can be used to improve the excitations effectiveness. In our study, this 
knowledge is given in terms of dimensional stability and control derivatives. Those parameters are used to obtain magnitude Bode plots that allow one to design an optimized input energy distribution. In order to achieve this aim, a genetic algorithm has bee used. The obtained non-uniform power spectrum has been used later to design multisine signals.

The model is excited with both sets of inputs, and the Sys-ID is performed for both cases separately. For estimating the stability and control derivatives, two methods are used: Equation Error and Output Error. The model parameters obtained from the OLS are biased as the approach is not resistant to the measurement noise in the independent variables. The second method allows one to obtain stability and control derivatives with a very high accuracy. On the basis of those outcomes, it is found that both manoeuvres allow one to obtain accurate estimates. Almost an ideal match between the results and measurements is observed. Introducing the $a$ priori information in the manoeuvre design enables evaluation of slightly better estimates.

However, when the initial knowledge of the system dynamics is not included, a slightly shorter time required for stabilization is observed. Nevertheless, in both cases the time that is required for performing the experiment is greatly reduced in comparison to the conventional approach, so both designs lower the overall cost of the Sys-ID process. The only noticeable drawback of the novel approach is that an additional time has to be spent on performing the energy distribution optimization.

Acknowledgement

This work has been supported by the Universidad de San Buenaventura in Colombia, Bogota and Deutsches Zentrum für Luft- und Raumfahrt e.V., Flugsystemtechnik Institut, Braunschweig, Germany.

\section{References}

1. Albisser M., Dobre S., Berner C., Thomassin M., Garnier H., 2017, Aerodynamic coefficient identification of a space vehicle from multiple free-flight tests, Journal of Spacecraft and Rockets, in press, DOI: 10.2514/1.A33587

2. Fedorov V.V., 1972, Theory of Optimal Experiments, Probability and Mathematical Statistics, Acade, New York, USA

3. Goodwin G.C., Payne R.L., 1977, Dynamic System Identification: Experiment Design and Data Analysis, Academic Press Inc., New York, USA

4. Grauer J.A., 2016, Aircraft fault detection using real-time frequency response estimation, AIAA Guidance, Navigation, and Control Conference, San Diego, USA, AIAA 20160372, DOI: 10.2514/6.2016-0372

5. Jategaonkar R.V., 2015, Flight Vehicle System Identification: A Time Domain Methodology, 2nd ed., Progess in Astronautics and Aeronautics, AIAA, Reston, USA, DOI: 10.2514/4.102790

6. Kalaba R.E., Springarn K., 1982, Control, Identification and Input Optimization, Mathematical Concepts and Methods in Science and Engineering, Plenum Press, New York, USA, DOI: 10.1007/978-1-4684-7662-0

7. Kendall M.G., Stuart A., Ord J.K., 1983, The Advanced Theory of Statistics, 4th ed., Vol. 3, Charles Griffin (Edit.), London, UK, DOI: 10.1002/for.3980040310

8. Lagarias J.C., Reeds J.A., Wright M.H., Wright P.E., 1998, Convergence properties of the Nelder-Mead simplex method in low dimensions, SIAM Journal of Optimization, 9, 1, 112147, DOI: $10.1137 / \mathrm{S} 1052623496303470$

9. Levin M.J., 1960, Optimum estimation of impulse response in the presence of noise, IRE Transactions on Circuit Theory, 7, 1, 5056, DOI: 10.1109/TCT.1960.1086622

10. Lichota P., 2016, Inclusion of the D-optimality in multisine manoeuvre design for aircraft parameter estimation, Journal of Theoretical and Applied Mechanics, 54, 1, 8798, DOI: 10.15632/jtam- pl.54.1.87 
11. Lichota P., Sibilski K., Ohme P., 2017, D-optimal simultaneous multistep excitations for aircraft parameter estimation, Journal of Aircraft, in press, DOI: 10.2514/1.C033794

12. Marchand M., 1974, Untersuchung der Bestimmbarkeit von Fleugzeugderivativen aus Flugversuchen, DLR, Report DFVLR-IB 154-74/32, Braunschweig, Germany

13. Morelli E.A., 2009, Flight test experiment design for characterizing stability and control of hypersonic vehicles, Journal of Guidance, Control and Dynamics, 32, 3, 949959, DOI: 10.2514/1.37092

14. Morelli E.A., Klein V., 1990, Optimal input design for aircraft parameter estimation using dynamic programming principles, AIAA Atmospheric Flight Mechanics Conference, Portland, USA, DOI: $10.2514 / 6.1990-2801$

15. Nahi N.E., Wallis D.E.J., 1969, Optimal inputs for parameter estimation in dynamic systems with white observation noise, Proceedings of Joint Automatic Control Conference, 506512

16. Schroeder M., 1970, Synthesis of low-peak-factor signals and binary sequences with low autocorrelation, IEEE Transactions on Information Theory, 16, 1, 8589, DOI: 10.1109/tit.1970.1054411

17. Seren C., Bommier F., Verdier L., Bucharles A., Alazard D., 2006, Optimal experiment and input design for flight test protocol optimization, AIAA Athmospheric Flight Mechanics Conference, Keystone, USA, DOI: 10.2514/6.2006-6280

18. Seren C., Hardier G., Roos C., 2013, Swarm intelligence and system identification: a hybrid discrete jumping frogs algorithm for optimal input design, 3rd IFAC International Conference on Intelligent Control, Chengdu, China

19. Tischler M.B., Remple R.K., 2012, Aircraft and Rotorcraft System Identification, 2nd ed., AIAA Education Series, AIAA, Washington D.C., USA, DOI: 10.2514/4.868207

20. VianA M.V.P., 2016, Time-domain system identification of rigid-body multipoint loads model, AIAA Atmospheric Flight Mechanics Conference, Washington D.C., USA, AIAA 20163706, DOI: 10.2514/6.2016-3706

21. Wells W.R., Ramachandran S., 1977, Multiple control input design for identification of light aircraft, IEEE Transactions on Automatic Control, 22, 985987, DOI: 10.1109/TAC.1977.1101653

22. Young P., Patton R.J., 1988, Frequency domain identification of remotely-piloted helicopter dynamics using frequency-sweep and Schroeder-phased test signals, AIAA Atmospheric Flight Mechanics Conference, Minneapolis, USA, AIAA 884349CP, DOI: 10.2514/6.1988-4349 